\title{
Cardiac and Peripheral Circulatory Responses to Angiotension and Vasopressin in Dogs
}

Richard W. Lee, Steven Standaert, Laryenth D. Lancaster, Donna Buckley, and Steven Goldman

Department of Internal Medicine, Tucson Veterans Administration Medical Center, Tucson, Arizona 85723; and University of Arizona, Tucson, Arizona 85724

\begin{abstract}
To determine the cardiac and peripheral circulatory responses to changes in afterload with angiotension and vasopressin, we increased mean aortic pressure $25 \%$ and $50 \%$ above control in splenectomized and ganglion-blocked dogs. We compared these responses to similar mechanical increases in aortic pressure produced by partial balloon occlusion of the descending aorta. With $25 \%$ or $50 \%$ increases in aortic pressure, angiotensin, vasopressin, and balloon inflation produced no changes in heart rate, right atrial, and mean pulmonary artery pressures. At $25 \%$ increase in aortic pressure, cardiac output was maintained with angiotensin and balloon occlusion but decreased with vasopressin. At $50 \%$ increase in aortic pressure, cardiac output was maintained with only balloon occlusion and decreased with both angiotensin and vasopressin. Whenever cardiac output fell, central blood volume did not increase as afterload increased. These changes in preload can be explained by alterations in the venous circulation. Vasopressin did not alter venous compliance or unstressed vascular volume but increased resistance to venous return. Angiotensin also increased resistance to venous return but decreased venous compliance and did not change unstressed vascular volume. Balloon occlusion had no effects on these parameters. We conclude that: $(a)$ angiotensin caused significant venoconstriction resulting in maintenance of cardiac output at $25 \%$ but not $50 \%$ increase in aortic pressure; $(b)$ vasopressin increased the resistance to venous return without venoconstriction; this resulted in a fall in cardiac output even with a $25 \%$ increase in aortic pressure; and (c) the effects of the agents on the venous circulation were independent of the mechanical effects of a pressure increase in the arterial circulation.
\end{abstract}

\section{Introduction}

It has been well established that forward flow, within physiologic limits, in the normal heart is not afterload dependent. Cardiac output is maintained with mechanical increases in mean aortic pressures up to $180 \mathrm{mmHg}$ (1). This information has to be reconciled with recent reports which show that humoral mediated increases in aortic pressure with angiotensin and vasopressin result in decreases in cardiac output $(2,3)$. A potential explanation for these differences may be the effects of these agents on the peripheral venous circulation. Changes in

Address reprint requests to Dr. Lee, Cardiology Section (111C), VA Medical Center, Tucson, AZ 85723.

Received for publication 8 September 1987 and in revised form 19 January 1988.

J. Clin. Invest.

(c) The American Society for Clinical Investigation, Inc.

0021-9738/88/08/0413/07 \$2.00

Volume 82, August 1988, 413-419 venous tone and the resulting blood volume shifts are known to alter cardiac output $(4,5)$.

The clinical importance of the effects of angiotensin and vasopressin have recently received attention because serum levels of these hormones are elevated in patients with congestive heart failure $(6,7)$. The increase in the levels of angiotensin and vasopressin are thought to be responsible for the generalized vasoconstriction in heart failure. Consequently, the use of specific antagonists as vasodilating agents to treat patients with heart failure has been advocated (8). To understand how these treatment regimens might affect patients, we have to first determine the complete consequences of humorally mediated vasoconstriction. Although the arterial constricting properties of these hormones are well appreciated, their effects on the venous circulation and subsequent volume shifts are poorly understood.

We have recently developed an experimental model in dogs to study the heart and peripheral circulation simultaneously and to examine volume shifts between the different cardiovascular compartments after an intervention (4). This is especially important when loading conditions of the heart are altered. In this model, splenectomies were performed to eliminate volume shifts that occur in dogs because the spleen is a large volume reservoir. Ganglion blockade was used to evaluate the direct effects of angiotensin and vasopressin independent of active reflexes.

The purpose of this study was to define the cardiac and peripheral circulatory effects of angiotensin and vasopressin. The effects of these hormones were compared to those resulting from mechanical increases in afterload produced by percutaneous placement of a balloon which partially occluded the descending aorta and increased aortic pressure. This mechanical increase in pressure was used to determine whether the alterations in the peripheral circulation were direct effects of these agents or were due to mechanical increases in afterload.

\section{Methods}

\section{Materials}

Seven mongrel dogs $(21 \pm 1 \mathrm{~kg})$ were used for the angiotensin and vasopressin studies. These animals were sedated with diazepam $(0.5$ $\mathrm{mg} / \mathrm{kg}$ ) and hydromorphone $(0.02 \mathrm{mg} / \mathrm{kg})$, anesthetized with halothane and nitrous oxide, and intubated for arterial and central line placements. A 7 French (Fr) thermodilution, flow-directed pulmonary artery catheter was inserted percutaneously in the right external jugular vein and advanced into the pulmonary artery. A $7 \mathrm{Fr}$ angiographic micromanometer tip catheter (Millar Instruments, Inc., Houston, TX) was inserted percutaneously via the left femoral artery for left ventricular and aortic pressure measurements. An additional 14-gauge plastic catheter was placed in the foreleg for intravenous access and blood volume exchanges. All animals were then placed on their left side on a warming blanket and extubated. Diazepam and hydromorphone were given for continued sedation and analgesia after recovery from general anesthesia. 


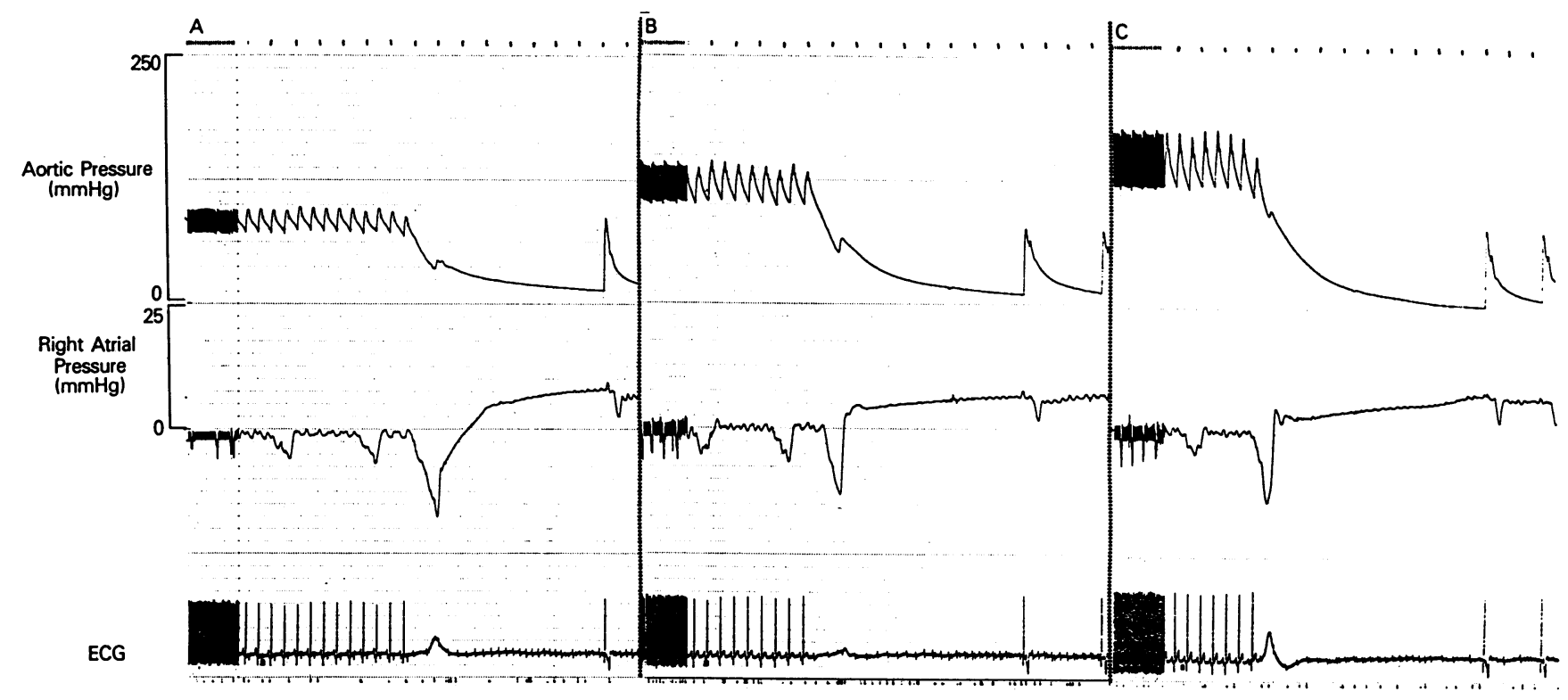

Figure 1. Measurement of MCFP using right atrial and arterial plateau pressures during balloon inflation in the descending aorta. $(A)$ Baseline. $(B)$ During balloon inflation to increase aortic pressure 25\%. (C) During balloon inflation to increase aortic pressure $50 \%$.
There is no change in the venous plateau pressure therefore no change in the calculated MCFP. Time lines on the top are 1s. (口) acetylcholine injection. ECG, electrocardiogram.
The pulmonary artery catheter was connected to a strain gauge pressure transducer (Statham 231D, Gould, Inc., Oxnard, CA) and calibrated with a mercury-filled manometer. The zero level was at a point on the animal's chest equal to $50 \%$ of the transthoracic diameter. The $7 \mathrm{Fr}$ Millar catheter tip pressure transducer was electronically zeroed and calibrated with a mercury manometer. The zero reference for the Millar catheter required placing the micromanometer in body temperature saline to obtain a stable zero reference. The data were recorded on an oscillographic recorder (ES 1000, Gould, Inc.). After all intravascular catheters were in place, ganglion blockade was induced with hexamethonium chloride $(30 \mathrm{mg} / \mathrm{kg})$. Control heart rate, pulmonary artery, pulmonary capillary wedge, right atrial, left ventricular, and aortic pressures were then recorded. Systemic vascular resistance was calculated as the difference between aortic and right atrial pressures divided by the cardiac output. Left ventricular $\mathrm{d} P / \mathrm{d} t$ was recorded through a Gould differentiator amplifier with input from the left ventricular pressure transducer. Cardiac output was obtained in triplicate by the thermodilution technique using a minicomputer (No. 9510-A, Edwards) and verified by the dye dilution technique during central blood volume determinations. Central blood volume, defined as the blood volume in the left side of the heart and lungs, was determined by the Stewart-Hamilton principle as previously described using indocyanine green dye (4). All blood withdrawn was immediately reinfused into the animals after sampling. Plasma volume was measured using the Evans Blue technique. A $0.45 \%$ (wt/vol) solution of dye in a total volume of $1 \mathrm{ml}$ was injected into the circulation via the external jugular vein sheath. $5 \mathrm{~min}$ after injection, an arterial blood sample of $7 \mathrm{ml}$ was withdrawn. After centrifugation, the dye concentration was determined spectrophotometrically. Total blood volume was calculated as: total blood volume $=$ plasma volume divided by $(1$ - hematocrit).

Mean circulatory filling pressure (MCFP) ${ }^{1}$ was obtained after transient asystole (5-10 s) was produced by a bolus injection of acetylcho-

1. Abbreviations used in this paper: APP, arterial plateau pressure; MCFP, mean circulatory filling pressure; VPP, venous plateau pressure. line (3-10 $\mathrm{mg}$ ) into the superior vena cava as previously described from our laboratory and others (Fig. 1) $(4,9,10)$. This pressure was calculated as: MCFP $=$ VPP + (APP - VPP $) \times$ arterial compliance/venous compliance, where VPP is the venous plateau pressure and APP is the arterial plateau pressure measured at $7 \mathrm{~s}$ after the start of asystole. An arterial-to-venous compliance ratio of 1:30 was assumed (4).

Resistance to venous return was calculated as the difference between MCFP and right atrial pressure divided by cardiac output.

Measurement of venous compliance. Venous compliance, defined as the change in volume per change in pressure of the total systemic vascular bed was determined by measurement of MCFP after acute volume changes. Compliances for control and after each intervention were measured on separate days.

In order to eliminate reflex sympathetic venoconstriction, ganglion blockade was indyced with hexamethonium chloride $(30 \mathrm{mg} / \mathrm{kg})$. Baseline compliance was determined over a range of physiologic venous pressures by measuring MCFP during transient circulatory arrests as previously described. This required repeated measurements of MCFP after volume expansion of $5 \mathrm{ml} / \mathrm{kg}$ with body temperature dextran and then $10 \mathrm{ml} / \mathrm{kg}$ with a combination of dextran and blood. All blood volume changes were performed by manual withdrawal and infusions within a 30-s period. After the MCFP was measured following the $5 \mathrm{ml} / \mathrm{kg}$ volume load with dextran, $5 \mathrm{ml} / \mathrm{kg}$ of blood was withdrawn and this plus another $5 \mathrm{ml} / \mathrm{kg}$ of dextran was used for the 10 $\mathrm{ml} / \mathrm{kg}$ volume load. There was at least a $15-\mathrm{min}$ recovery period between circulatory arrests to allow hemodynamic values to return to baseline. Venous compliance was defined as the reciprocal of the slope of the line obtained by plotting MCFP vs. blood volume. Unstressed vascular volume or the volume in the blood vessels at zero transmural pressure was obtained by extrapolating this pressure-volume relationship to the zero pressure intercept.

Measurement of arterial compliance. The arterial compliance was measured based on the simple Windkessel model of the circulation. Equations used were obtained from an electrical analogue of the RC circuit $(11,12)$. This model assumes that the arterial pressure initially decays monoexponentially as a function of time from an initial driving pressure with a rate constant $k_{3}$.

$P(t)=k_{1}+k_{2} e^{-k_{3} t}$ 
where $k_{1}$ is the APP (mmHg), $k_{2}$ is the arterial driving pressure (mm $\mathrm{Hg}$ ), and $P(t)$ is the instantaneous pressure $(\mathrm{mmHg})$.

Arterial compliance was then calculated:

$T=C R$

where $T$ is the time constant, $C$ is compliance $(\mathrm{ml} / \mathrm{mmHg})$, and $R$ is the systemic vascular resistance $(\mathrm{mmHg} \mathrm{min} / \mathrm{kg} \cdot \mathrm{ml})$.

The aortic pressure recorded during acetylcholine-induced circulatory arrests was digitized and entered into the computer (model 2100, Hewlett Packard Co., Palo Alto, CA). According to Eq. 1, the time constant is inversely proportional to the slope of a plot of $\ln$ pressure vs. time. Compliance was calculated from Eq. 2 . The volume of blood shifted into or out of the arterial system is equal to the product of arterial compliance and the change in mean aortic pressure (12).

\section{Experimental protocol}

After baseline studies were performed including measurements of hemodynamics, MCFP, venous and arterial compliances, angiotensin $(0.01-0.02 \mu \mathrm{g} / \mathrm{kg} \cdot \mathrm{min})$ and vasopressin $(3.5-5.0 \mathrm{ng} / \mathrm{kg} \cdot \mathrm{min})$ were infused in random order to increase arterial pressure $25 \%$ above control. When the pressure was stable for $15 \mathrm{~min}$, repeat hemodynamics including MCFP were measured. Repeat baseline values including MCFP were obtained and then in random order, angiotensin (0.03$0.05 \mu \mathrm{g} / \mathrm{kg} \cdot \mathrm{min})$ or vasopressin $(7.0-10.0 \mathrm{ng} / \mathrm{kg} \cdot \min )$ as infused to increase arterial pressure $50 \%$ above control. When the pressure was stable for $15 \mathrm{~min}$, repeat measurements of hemodynamics were done. During the $50 \%$ increase in aortic pressure venous and arterial compliances were measured as described above. Each intervention value was compared to its respective baseline.

In four separate dogs aortic pressure was increased $25 \%$ and $50 \%$ mechanically. Experiments were performed after splenectomy and the dogs were instrumented as described above. Ganglion blockade was achieved with hexamethonium chloride $(30 \mathrm{mg} / \mathrm{kg})$. In addition, an arterial cutdown was performed on the right carotid and right and left femoral arteries. Under fluoroscopic guidance a $7 \mathrm{Fr}$ micrommanometer catheter was placed in the left ventricle. A $25-\mathrm{mm}$ balloon catheter specially designed (Datascope Inc., Oakland, NJ) was advanced through the left femoral artery into the descending aorta. Through the right femoral artery a 5 Fr pigtail and 5 Fr micromanometer tip catheter were placed in the ascending aorta, proximal to the balloon, for aortic pressure and central blood volume measurements. The experimental protocol was similar to the studies described for angiotensin and vasopressin. Mechanical hypertension was produced by inflating the balloon with normal saline to increase arterial pressure $25 \%$ and $50 \%$ above control. Baseline hemodynamics, total and central blood volumes, and MCFP were measured. Mean aortic pressure was then increased $25 \%$ above baseline. After $15 \mathrm{~min}$ of stable pressures all measurements were repeated. Mean aortic pressure was then increased
50\% above baseline. Again, after 15 min all measurements were obtained. In addition, at $50 \%$ increase in pressure arterial and venous compliances were obtained as described above.

This protocol was approved by the animal research committees of the Tucson Veterans Administration Hospital and the University of Arizona. Specific attention was given to the appropriateness and welfare of the animal model, the adequacy of anesthesia and to the methods of instrumentation. During the measurement of MCFP, cardiac electrical activity resumed spontaneously within $10 \mathrm{~s}$ in all animals, no seizures occurred and there was no evidence of pain or suffering as defined in the Federal Animal Welfare Act and in accordance with the National Institutes of Health Guide for care and use of laboratory animals.

\section{Statistical analysis}

Data are expressed as mean \pm standard error of the mean. Student's $t$ test for paired values was used to compare each intervention with the appropriate baseline value. Repeated measure analysis of variance was used to compare serial measurements to control with the balloon inflation. To compare values between groups, analysis of variance was used. Venous compliance was determined by regression analysis using the method of least squares.

\section{Results}

Effects of angiotensin on hemodynamics. The changes in hemodynamics resulting from $25 \%$ and $50 \%$ increases in mean aortic pressure with angiotensin are shown in Table $I$. With a $25 \%$ or $50 \%$ increase there were no changes in heart rate, right atrial, and pulmonary artery pressures. With a $25 \%$ increase, left ventricular end-diastolic pressure increased from $3.6 \pm 0.9$ to $5.9 \pm 1.0 \mathrm{mmHg}(P<0.02), \mathrm{d} P / \mathrm{d} t$ increased $10 \%(P<0.02)$, and systemic vascular resistance increased from $0.49 \pm 0.02$ to $0.61 \pm 0.03 \mathrm{mmHg} \mathrm{min} / \mathrm{kg} \cdot \mathrm{ml}(P<0.01)$ with no changes in cardiac output. With a $50 \%$ increase in aortic pressure, left ventricular end-diastolic pressure increased to $10.5 \pm 1.6$ $\mathrm{mmHg}(P<0.01)$, left ventricular $\mathrm{d} P / \mathrm{d} t$ increased $17 \%(P$ $<0.01)$ while cardiac output decreased $9.3 \%(P<0.01)$. The result was an increase in systemic vascular resistance. Thus, with the $50 \%$ increase in aortic pressure there was a fall in cardiac output compared with no change with the $25 \%$ increase in pressure.

Effects of vasopressin on hemodynamics. The changes in hemodynamics resulting from $25 \%$ and $50 \%$ increases in aortic pressure are shown in Table II. With a $25 \%$ or $50 \%$ increase there were no changes in heart rate, right atrial, and pulmo-

Table I. Hemodynamic Changes Resulting from an Infusion of Angiotensin, 0.01-0.02 and 0.03-0.05 $\mu \mathrm{g} / \mathrm{kg} \cdot \mathrm{min}$, to Increase Mean Aortic Pressure 25\% and 50\% above Baseline during Ganglion Blockade with Hexamethonium

\begin{tabular}{|c|c|c|c|c|}
\hline & Baseline & Angiotensin (25\%) & Baseline & Angiotensin (50\%) \\
\hline Heart rate (beats/min) & $102 \pm 2$ & $104 \pm 4$ & $104 \pm 5$ & $109 \pm 4$ \\
\hline Mean aortic pressure $(\mathrm{mmHg})$ & $76 \pm 4$ & $96 \pm 6^{8}$ & $81 \pm 3$ & $125 \pm 3^{8}$ \\
\hline $\mathrm{LV} \mathrm{d} P / \mathrm{d} t(m m H g / s)$ & $1693 \pm 85$ & $1836 \pm 85^{*}$ & $1643 \pm 95$ & $1956 \pm 47^{\ddagger}$ \\
\hline LV systolic pressure $(\mathrm{mmHg})$ & $95 \pm 5$ & $111 \pm 6^{\ddagger}$ & $100 \pm 4$ & $151 \pm 5^{8}$ \\
\hline $\mathrm{LV}$ end-diastolic pressure $(\mathrm{mmHg})$ & $3.6 \pm 0.9$ & $5.9 \pm 1.0^{*}$ & $5.0 \pm 0.7$ & $10.5 \pm 1.6^{ \pm}$ \\
\hline Right atrial pressure $(\mathrm{mmHg})$ & $0.8 \pm 0.5$ & $0.6 \pm 0.7$ & $0.9 \pm 0.7$ & $0.2 \pm 0.8$ \\
\hline Mean pulmonary artery pressure $(\mathrm{mmHg})$ & $11 \pm 1$ & $13 \pm 1$ & $12 \pm 1$ & $13 \pm 1$ \\
\hline Cardiac index $(\mathrm{ml} / \mathrm{kg} \cdot \mathrm{min})$ & $156 \pm 10$ & $157 \pm 4$ & $161 \pm 8$ & $146 \pm 8^{\ddagger}$ \\
\hline Systemic vascular resistance $(\mathrm{mmHg} \mathrm{min} / \mathrm{kg} \cdot \mathrm{ml})$ & $0.49 \pm 0.2$ & $0.61 \pm 0.03^{\ddagger}$ & $0.49 \pm 0.03$ & $0.85 \pm 0.04^{8}$ \\
\hline
\end{tabular}

Values are mean $\pm \mathrm{SE}$ in seven splenectomized dogs. ${ }^{*} P<0.02{ }^{\ddagger} P<0.01{ }^{8} P<0.001$ compared to baseline. LV, left ventricular. 
Table II. Hemodynamic Changes Resulting from an Infusion of Vasopressin, 3.5-5.0 and 7.0-10.0 ng/ $\mathrm{kg} \cdot \mathrm{min}$, to Increase Mean Aortic Pressure 25\% and 50\% above Baseline during Ganglion Blockade with Hexamethonium

\begin{tabular}{|c|c|c|c|c|}
\hline & Baseline & Vasopressin (25\%) & Baseline & Vasopressin (50\%) \\
\hline Heart rate (beats/min) & $103 \pm 6$ & $96 \pm 6$ & $102 \pm 6$ & $98 \pm 5$ \\
\hline Mean aortic pressure $(\mathrm{mmHg})$ & $69 \pm 2$ & $86 \pm 1^{8}$ & $72 \pm 6$ & $110 \pm 8^{8}$ \\
\hline $\mathrm{LV} \mathrm{d} P / \mathrm{d} t(m m H g / s)$ & $1564 \pm 71$ & $1707 \pm 70^{\ddagger}$ & $1586 \pm 79$ & $1821 \pm 94^{*}$ \\
\hline LV systolic pressure $(\mathrm{mmHg})$ & $92 \pm 2$ & $107 \pm 2^{8}$ & $92 \pm 6$ & $134 \pm 9^{8}$ \\
\hline LV end-diastolic pressure $(\mathrm{mmHg})$ & $3.6 \pm 0.6$ & $5.6 \pm 0.8^{\ddagger}$ & $1.3 \pm 0.6$ & $7.1 \pm 1.4^{*}$ \\
\hline Right atrial pressure $(\mathrm{mmHg})$ & $-0.2 \pm 0.2$ & $0.0 \pm 0.1$ & \pm 0.8 & $0.3 \pm 0.5$ \\
\hline Mean pulmonary artery pressure $(\mathrm{mm} \mathrm{Hg})$ & $14 \pm 12$ & $15 \pm 1$ & $11 \pm 1$ & $12 \pm 1$ \\
\hline Cardiac index $(\mathrm{ml} / \mathrm{kg} \cdot \mathrm{min})$ & $150 \pm 11$ & $124 \pm 7^{\ddagger}$ & $149 \pm 7$ & $120 \pm 4^{*}$ \\
\hline Systemic vascular resistance $(\mathrm{mmHg} \mathrm{min} / \mathrm{kg} \cdot \mathrm{ml})$ & $0.48 \pm 0.04$ & $0.71 \pm 0.05^{\S}$ & $0.49 \pm 0.03$ & $0.92 \pm 0.05^{\S}$ \\
\hline
\end{tabular}

Values are mean $\pm \mathrm{SE}$ in seven splenectomized dogs. ${ }^{*} P<0.02 ;{ }^{\ddagger} P<0.01 ;{ }^{\S} P<0.001$ compared to baseline. LV, left ventricular.

nary artery pressures. With a $25 \%$ increase in aortic pressure, left ventricular end-diastolic pressure increased from $3.5 \pm 0.7$ to $5.6 \pm 0.8 \mathrm{mmHg}(P<0.01)$ and $\mathrm{d} P / \mathrm{d} t$ increased $8.4 \%(P$ $<0.01)$. Cardiac output decreased $19 \%(P<0.01)$. The result was an increase in systemic vascular resistance from $0.48 \pm 0.05$ to $0.71 \pm 0.05 \mathrm{mmHg} \mathrm{min} / \mathrm{kg} \cdot \mathrm{ml}(P<0.001)$. With a $50 \%$ increase in aortic pressure, left ventricular end-diastolic pressure increased to $7.1 \pm 1.4 \mathrm{mmHg}(P<0.02)$, left ventricular $\mathrm{d} P / \mathrm{d} t$ increased $17 \%(P<0.02)$, while cardiac output decreased 22\% $(P<0.02)$. The result was an increase in systemic vascular resistance. Thus, there was a decrease in cardiac output with both the $25 \%$ and $50 \%$ increase in aortic pressure.

Effects of angiotensin on the arterial and venous circulations. There was a graded increase in MCFP during both increases in pressure (Tables III and IV). Venous compliance decreased from $2.0 \pm 0.1$ to $1.5 \pm 0.1 \mathrm{ml} / \mathrm{mmHg} \cdot \mathrm{kg}(P<0.01)$. The correlation coefficients for each of the MCFP-blood volume relationships were $r=0.999$ and $r=0.996$, respectively. Resistance to venous return increased with both increases in aortic pressure. The shift in venous compliance was not associated with any changes in total blood volume or unstressed vascular volume. Central blood volume increased from a baseline value of $20 \pm 1$ to $25 \pm 1 \mathrm{ml} / \mathrm{kg}(P<0.001)$ with $25 \%$ and to $24 \pm 1 \mathrm{ml} / \mathrm{kg}(P<0.01)$ with the $50 \%$ increase in aortic pres-

Table III. Changes in MCFP, Blood Volume Distribution, Arterial, and Venous Compliances during an Infusion of Angiotensin, 0.01-0.02 $\mu \mathrm{g} / \mathrm{kg} / \mathrm{min}$, to Increase Mean Aortic Pressure 25\% above Baseline during Ganglion Blockade with Hexamethonium

\begin{tabular}{lcc}
\hline & Baseline & Angiotensin (25\%) \\
\hline MCFP $(\mathrm{mmHg})$ & $6.1 \pm 0.3$ & $7.4 \pm 0.3^{\S}$ \\
Resistance to venous return & & \\
$\quad(\mathrm{mmHg} \mathrm{min} / \mathrm{kg} \cdot \mathrm{ml})$ & $0.045 \pm 0.003$ & $0.052 \pm 0.004^{*}$ \\
Total blood volume $(\mathrm{ml} / \mathrm{kg})$ & $77 \pm 1$ & $76 \pm 2$ \\
Central blood volume $(\mathrm{ml} / \mathrm{kg})$ & $20 \pm 1$ & $25 \pm 1^{\S}$ \\
Arterial compliance $(\mathrm{ml} / \mathrm{mmHg} \cdot \mathrm{kg})$ & $0.072 \pm 0.004$ & $0.060 \pm 0.003^{\ddagger}$ \\
Arterial volume shift $(\mathrm{ml} / \mathrm{kg})$ & & $1.1 \pm 0.1$
\end{tabular}

Values are mean $\pm \mathrm{SE}$ in seven splenectomized dogs.

* $P<0.02 ;^{\ddagger} P<0.01 ;^{8} P<0.001$ compared to baseline values. sure. In both instances this represented a $25 \%$ increase from baseline in spite of the differences in afterload. Arterial compliance decreased with the increase in aortic pressure. Thus the major change in the venous circulation was venoconstriction manifested as a change in venous compliance.

Effects of vasopression on the arterial and venous circulations. There were small increases in MCFP during both increases in pressure (Tables V and VI). Venous compliance did not change in spite of the $50 \%$ increase in aortic pressure, $2.0 \pm 0.1$ vs. $1.8 \pm 0.1 \mathrm{ml} / \mathrm{mmHg} \cdot \mathrm{kg}$. The correlation coefficients for each of the MCFP-blood volume relationships were $r$ $=0.999$ and $r=0.997$, respectively. Resistance to venous return increased with both infusions. These changes were not associated with any changes in total blood volume, unstressed vascular volume, and central blood volume. Arterial compliance decreased with the increase in aortic pressure. In summary, vasopressin increased resistance to venous return with no venoconstriction.

Comparison of angiotensin, vasopressin, and balloon occlusion. With all three interventions, there were comparable changes in aortic pressure (Fig. 2). The changes in cardiac output, MCFP, venous compliance, and central blood volume

Table IV. Changes in MCFP, Blood Volume Distribution, Arterial, and Venous Compliances during an Infusion of Angiotensin, 0.03-0.05 $\mu \mathrm{g} / \mathrm{kg} \cdot \mathrm{min}$, to Increase Mean Aortic Pressure 50\% above Baseline during Ganglion Blockade with Hexamethonium

\begin{tabular}{lcc}
\hline & Baseline & Angiotensin (50\%) \\
\hline MCFP $(\mathrm{mmHg})$ & $7.1 \pm 0.4$ & $9.4 \pm 0.3^{8}$ \\
Venous compliance $(\mathrm{ml} / \mathrm{mmHg} \cdot \mathrm{kg})$ & $2.0 \pm 0.1$ & $1.5 \pm 0.1^{\ddagger}$ \\
Unstressed vascular volume $(\mathrm{ml} / \mathrm{kg})$ & $64.2 \pm 6.4$ & $64.2 \pm 7.9$ \\
Resistance to venous return & & \\
$\quad(\mathrm{mmHg}$ min $/ \mathrm{kg} \cdot \mathrm{ml})$ & $0.051 \pm 0.003$ & $0.076 \pm 0.006^{\ddagger}$ \\
Total blood volume $(\mathrm{ml} / \mathrm{kg})$ & $78 \pm 2$ & $77 \pm 2$ \\
Central blood volume $(\mathrm{ml} / \mathrm{kg})$ & $19 \pm 1$ & $23 \pm 1^{\S}$ \\
Arterial compliance $(\mathrm{ml} / \mathrm{mmH} \cdot \mathrm{kg})$ & $0.070 \pm 0.006$ & $0.045 \pm 0.002^{\ddagger}$ \\
Arterial volume shift $(\mathrm{ml} / \mathrm{kg})$ & & $2.0 \pm 0.1$
\end{tabular}

Values are mean $\pm \mathrm{SE}$ in seven splenectomized dogs.

${ }^{\ddagger} P<0.01 ;{ }^{\S} P<0.001$ compared to baseline values. 
Table V. Changes in MCFP, Blood Volume Distribution, Arterial, and Venous Compliances during an Infusion of Vasopressin, 3.5-5.0 $\mathrm{ng} / \mathrm{kg} \cdot \mathrm{min}$, to Increase Mean Aortic Pressure 25\% above Baseline during Ganglion Blockade with Hexamethonium

\begin{tabular}{lcc}
\hline & Baseline & Vasopressin (25\%) \\
\hline MCFP $(\mathrm{mmHg})$ & $6.9 \pm 0.4$ & $7.4 \pm 0.3$ \\
Resistance to venous return $(\mathrm{mmHg}$ & & \\
$\quad \mathrm{min} / \mathrm{kg} \cdot \mathrm{ml})$ & $0.047 \pm 0.005$ & $0.059 \pm 0.004^{\S}$ \\
Total blood volume $(\mathrm{ml} / \mathrm{kg})$ & $79 \pm 3$ & $78 \pm 3$ \\
Central blood volume $(\mathrm{ml} / \mathrm{kg})$ & $20 \pm 1$ & $19 \pm 1$ \\
Arterial compliance $(\mathrm{ml} / \mathrm{mmHg} \cdot \mathrm{kg})$ & $0.080 \pm 0.006$ & $0.048 \pm 0.003^{\S}$ \\
Arterial volume shift $(\mathrm{ml} / \mathrm{kg})$ & & $0.8 \pm 0.1$ \\
\hline
\end{tabular}

Values are mean $\pm \mathrm{SE}$ in seven splenectomized dogs.

${ }^{*} P<0.02 ;{ }^{\S} P<0.001$ compared to baseline values.

are compared among the three interventions in Fig. 2. At a $50 \%$ increase in aortic pressure, cardiac output decreased with both angiotensin and vasopressin but did not change with balloon occlusion. Mean circulatory filling pressure increased and venous compliance decreased only with angiotensin. Central blood volume increased at the $50 \%$ increase in aortic pressure with the balloon but not with angiotensin or vasopressin. In summary, with comparable increases in mean aortic pressure cardiac output was maintained only with balloon occlusion because adequate preload resulted from an appropriate volume shift into the central compartment.

\section{Discussion}

The results of this study show that when afterload was increased mechanically, cardiac output was maintained. When afterload was increased with angiotensin or vasopressin, cardiac output decreased depending on the elevation of aortic

Table VI. Changes in MCFP, Blood Volume Distribution, Arterial, and Venous Compliances during an Infusion of Vasopressin, 7.0-10.0 $\mathrm{ng} / \mathrm{kg} \cdot \mathrm{min}$, to Increase Mean Aortic Pressure 50\% above Baseline during Ganglion Blockade with Hexamethonium

\begin{tabular}{lcc}
\hline & Baseline & Vasopressin (50\%) \\
\hline MCFP $(\mathrm{mmHg})$ & $6.8 \pm 0.2$ & $7.6 \pm 0.2$ \\
Venous compliance $(\mathrm{ml} / \mathrm{mmHg} \cdot \mathrm{kg})$ & $2.0 \pm 0.1$ & $1.8 \pm 0.1$ \\
Unstressed vascular volume $(\mathrm{ml} / \mathrm{kg})$ & $64.2 \pm 6.4$ & $65.8 \pm 8.5$ \\
Resistance to venous return & & \\
$\quad(\mathrm{mmHg} \mathrm{min} / \mathrm{kg} \cdot \mathrm{ml})$ & $0.044 \pm 0.001$ & $0.064 \pm 0.002^{\ddagger}$ \\
Total blood volume $(\mathrm{ml} / \mathrm{kg})$ & $77 \pm 2$ & $78 \pm 2$ \\
Central blood volume $(\mathrm{ml} / \mathrm{kg})$ & $21 \pm 1$ & $21 \pm 1$ \\
Arterial compliance $(\mathrm{ml} / \mathrm{mmHg} \cdot \mathrm{kg})$ & $0.064 \pm 0.009$ & $0.044 \pm 0.002^{*}$ \\
Arterial volume shift $(\mathrm{ml} / \mathrm{kg})$ & & $1.7 \pm 0.1$ \\
& & \\
\hline
\end{tabular}

Values are mean $\pm \mathrm{SE}$ in seven splenectomized dogs.

${ }^{*} P<0.02 ;{ }^{\ddagger} P<0.01$ compared to baseline values.

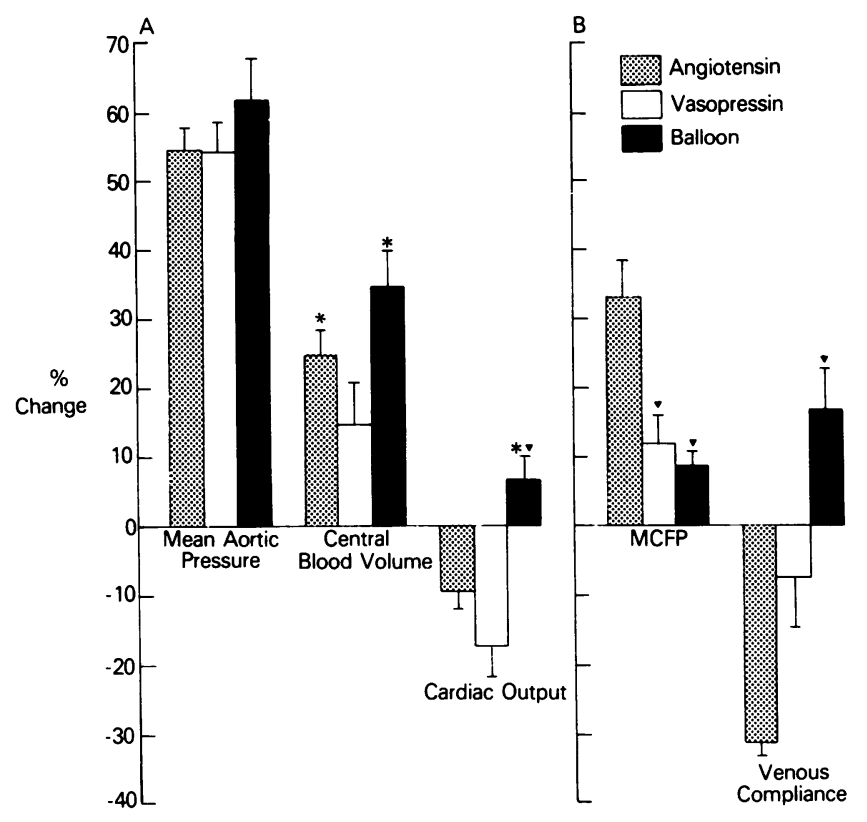

Figure 2. Comparison among angiotensin, vasopressin, and balloon occlusion. $(A)$ Bar graphs comparing percent changes above/below control in aortic pressure, cardiac output, and central blood volume during a $50 \%$ increase in mean aortic pressure with angiotensin, vasopressin, and balloon occlusion. Central blood volume increased significantly for both angiotensin and balloon occlusion compared to vasopressin. Cardiac output decreased with angiotensin and vasopressin but was maintained with balloon occlusion. (*) $P<0.01$ vs. vasopressin; $(\nabla) P<0.01$ vs. angiotensin. $(B)$ Percent change in MCFP and venous compliance with a $50 \%$ increase in aortic pressure with angiotensin, vasopressin, and balloon occlusion. MCFP increased more with angiotensin when compared to vasopressin and balloon occlusion. Venous compliance decreased significantly with angiotensin when compared to balloon occlusion. (v) $P<0.01$ vs. angiotensin.

pressure. With a $25 \%$ increase in aortic pressure, cardiac output decreased with vasopressin but was maintained with angiotensin. With a $50 \%$ increase in aortic pressure cardiac output fell with both agents. These different effects on cardiac output can be explained by changes in the venous circulation. Angiotensin decreased venous compliance with no change in unstressed vascular volume. Resistance to venous return increased with both increases in afterload but venoconstriction was adequate to increase preload and maintain forward flow with the $25 \%$ and not the $50 \%$ increase in aortic pressure. Vasopressin did not change either venous compliance or unstressed vascular volume but resistance to venous return increased with both a $25 \%$ and $50 \%$ elevation of aortic pressure. This resulted in a fall in cardiac output with both levels of increase in afterload. These alterations in the peripheral circulation were not seen with mechanical increases in aortic pressure produced by balloon inflation and therefore represent the direct effects of these agents on the venous circulation.

The effects of angiotensin and vasopressin on the heart and peripheral circulation have recently received special attention because investigators have reported elevated levels of these hormones in patients with congestive heart failure (6). The vasoconstriction resulting from these and other hormones, like norepinephrine, is thought to be responsible for the increased 
afterload in these patients. Because of clinical investigations that focus on the use of vasodilating agents to decrease afterload, vasodilating agents have become the mainstay of therapy for patients in heart failure $(8,13)$.

The present study was performed to evaluate how increases in afterload produced by an increase in aortic pressure with these agents may affect the cardiovascular system. Specifically, we focused on the effects of these agents on the venous circulation. In clinical studies, changes in central venous pressure have been equated with changes in venous tone. However central venous pressure is affected by heart rate, chamber stiffness, afterload, and contractility and does not reflect changes in the venous capacitance system. With the methods used in the present study, changes in the venous capacitance system were obtained by measuring mean circulatory filling pressure, vascular compliance, and unstressed vascular volume. These concepts and their role in the regulation of cardiac output have been reviewed $(14,15)$.

The changes in baseline hemodynamics with angiotensin are consistent with previous reports (3). As aortic pressure increased, there was a tendency for heart rate to increase but this was not statistically significant. Although left ventricular $\mathrm{d} P / \mathrm{d} t$ increased, this was probably the result of an increase in afterload and not an increase in intropic state because $\mathrm{d} P / \mathrm{d} t$ also increased with vasopressin and balloon inflation. In addition, previous investigators have shown that angiotensin does not shift the end-systolic pressure-volume relationship (2). Mean circulatory filling pressures and resistance to venous return increased with both increases in aortic pressure. However, the venoconstriction with a $25 \%$ increase in aortic pressure was adequate to maintain cardiac output and stroke volume. Venoconstriction probably occurred from an increase in smooth muscle tone. This resulted in a decrease in venous compliance with no change in unstressed vascular volume. Central blood volume and left ventricular end-diastolic pressure increased. With greater increases in aortic pressure (50\%) the venoconstricting effects of angiotensin were not adequate to overcome the increase in resistance to venous return. The result was no additional increase in central blood volume or preload, so cardiac output decreased because of the preloadafterload mismatch $(2,9)$.

Increases in afterload with vasopressin caused no significant change in right atrial, mean pulmonary artery and pulmonary artery wedge pressures. Previous workers have shown that in reflex intact animals, vasopressin infusions will not increase aortic pressure $(16,17)$. Because our animals were ganglion blocked, an increase in aortic pressure was easily achieved. Similarly the slight but not significant fall in heart rate may represent a direct effect on the heart. In contrast to angiotensin, with both vasopressin infusion doses there was a significant decrease in cardiac output and no change in central blood volume. Similar decreases in cardiac output with vasopressin have been reported by others $(16,17)$. The fall in cardiac output can be explained by the effects of vasopressin on the peripheral circulation. Vasopressin produced no significant changes in mean circulatory filling pressure, venous compliance and unstressed vascular volume. This coupled with the increase in resistance to venous return and afterload, resulted in a fall in cardiac output even with a $25 \%$ increase in aortic pressure (18).

To evaluate the possibility that these peripheral circulatory changes with angiotensin and vasopressin may have been secondary to the increase in aortic pressure we produced me- chanical arterial hypertension and repeated the same studies. A specially designed balloon catheter, placed percutaneously in the descending aorta above the splanchnic bed, was used to increase mean aortic pressure. With mechanical hypertension cardiac output was maintained, while central blood volume increased. There were no changes in any of the venous circulation parameters including resistance to venous return. Our data are consistent with the classical studies of Herndon and Sagawa (1) and Stokland et al. (19, 20). These investigators showed that cardiac output was independent of afterload up to a mean aortic pressure of $180 \mathrm{mmHg}$ (1). As afterload increases there is a redistribution of cardiac output and left ventricular filling is maintained (19). Left ventricular end-diastolic volume increases and the ventricle shifts to the plateau of the Frank-Starling curve. With reflexes intact any increase in aortic pressure will cause an increase in venous capacitance. This did not occur in our study with the mechanical increase in pressure because reflexes were blocked with hexamethonium. An alternative explanation, not addressed in this study, involves the effects on the splanchnic circulation, its role as a volume reservoir, and changes in portal pressure (18). Although investigators have shown that portal pressure does not equilibrate with MCFP, the directional changes were the same with volume loading and depletion (21). With balloon inflation in the descending aorta there is a decrease in flow to the splanchnic bed. This results in a passive redistribution of volume from the splanchnic bed into the central compartment maintaining preload and cardiac output. With angiotensin infusion there is active venous constriction and an increase in portal pressure so that there is no passive splanchnic redistribution of volume. This results in a maintenance of cardiac output with $25 \%$ but a decrease with $50 \%$ increase in aortic pressure. Vasopressin causes no change in portal pressure and no venoconstriction so that cardiac output falls under both conditions.

Another potential explanation for the effects of these three interventions on the venous circulation may be due to differential effects on systemic and splanchnic beds. Previous investigators have shown that the circulation can be divided into at least two beds with different time constants of drainage (22). A redistribution of cardiac output away from the splanchnic bed or long time-constant system would result in passive drainage of blood to maintain cardiac output $(23,24)$.

All of these changes in the venous system occurred with similar effects of these interventions on the arterial circulation. In each instance the increase in aortic pressure was associated with a decrease in arterial compliance and volume shifts into the arterial circulation depending on the level of the increase in pressure. Since the arterial circulation contains a relatively small amount of blood compared to the venous circulation, it is difficult to imagine that changes in the arterial system are responsible for the blood volume shifts that determine preload. It should be emphasized that these studies were performed in ganglion-blocked dogs with normal cardiac and peripheral circulatory hemodynamics. In the presence of congestive heart failure with intact reflexes the effects may be different.

In conclusion, we have defined the effects of angiotensin and vasopressin on the heart and the peripheral circulation. These changes in the peripheral circulation can be used to explain the changes in cardiac output seen with the infusion of these agents. Finally, these peripheral circulatory changes are independent of the mechanical effects of a pressure increase in the arterial circulation. 


\section{Acknowledgments}

The authors would like to thank James Nelson and James Thome for their technical assistance and Datascope Inc., Houston, TX for supplying the intraaortic balloon.

\section{References}

1. Herndon, C. W., and K. Sagawa. 1969. Combined effects of aortic and right atrial pressures on aortic flow. Am. J. Physiol. 217:65-72.

2. Lee, J. D., T. Tajimi, J. Patritti, and J. Ross. 1986. Preload reserve and mechanisms of afterload mismatch in normal conscious dogs. Am. J. Physiol. 250:H464-H473.

3. Heyndrickx, G. R., D. H. Boettcher, and S. F. Vatner. 1976. Effects of angiotensin, vasopressin and methoxamine on cardiac function and blood flow distribution in conscious dogs. Am. J. Physiol. 213:1579-1587.

4. Lee, R. W., R. G. Gay, L. D. Lancaster, M. Olajos, and S. Goldman. 1987. Dog model to study the effects of pharmacologic agents on the peripheral circulation: effects of milrinone. J. Pharmacol. Exp. Ther. 240:1014-1019.

5. Guyton, A. C., C. G. Jones, and T. G. Coleman. 1973. Cardiac Output and its Regulation. W. B. Saunders Co., Philadelphia. 173-233.

6. Dzau, V. J., W. S. Colucci, N. K. Hollenberg, and G. H. Williams. 1981. Relation of the renin-angiotensin-aldosterone system to clinical state in congestive heart failure. Circulation. 63:645-651.

7. Szatalowicz, V. L., P. E. Arnold, C. Chaimowitz, D. Bichet, T. C. Berl, and R. W. Schrier. 1981. Radioimmunoassay of plasma arginine vasopressin in hyponatremic patients with congestive heart failure. $N$. Engl. J. Med. 305:263-266.

8. The CONSENSUS Trial Study Group. 1987. Effects of enalapril on mortality in severe congestive heart failure. N. Engl. J. Med. 316:1429-1435.

9. Lee, R. W., L. D. Lancaster, D. Buckley, and S. Goldman. 1987. Peripheral circulatory control of preload-afterload mismatch with angiotensin in dogs. Am. J. Physiol. 253:H126-132.

10. Young, B. D., R. H. Murray, R. G. Bengis, and A. K. Markov. 1980. Experimental angiotensin II hypertension. Am. J. Physiol. 239:H391-H398.

11. Simon, A. C., M. E. Safar, J. A. Levenson, G. M. London, B. I.
Levy, and N. P. Chau. 1979. An evaluation of large arteries compliance in man. Am. J. Physiol. 237:H550-H554.

12. Stein, P. M., C. L. MacAnespie, and C. F. Rothe. 1983. Total body vascular capacitance changes during high intercranial pressure in dogs. Am. J. Physiol. 245:H947-H956.

13. Cohn, J. N., D. Archibald, S. Zieshe, J. A. Franciosa, W. E. Houston, F. Tristani, B. Dinkman, W. Jacobs, G. Francis, K. Flohr, S. Goldman, F. Cobb, P. Shah, R. Saunders, R. Fletcher, H. Loeb, V. Hughes, and B. Baker. 1986. Effects of vasodilator therapy on mortality in chronic congestive heart failure. N. Engl. J. Med. 314:15471553.

14. Rothe, C. F. 1983. Venous system: physiology of the capacitance vessels. In Handbook Physiol. Sect. 2: The Cardiovascular System. William \& Wilkins Co., Baltimore, MD. 397-452.

15. Greenway, C. V., and W. W. Lautt. 1986. Blood volume, the venous system, preload and cardiac output. Can. J. Physiol. Pharmacol. 64:383-387.

16. Brown, A. J., T. E. Lohmeier, R. G. Carroll, and E. F. Meydrech. 1986. Cardiovascular and renal responses to chronic vasopressin infusion. Am. J. Physiol. 250:H584-594.

17. Webb, R. L., J. W. Osborn, and A. W. Cowley. 1986. Cardiovascular actions of vasopressin: baroreflex modulation in the conscious rat. Am. J. Physiol. 251:H1244-1251.

18. Greenway, C. V., K. L. Seaman, and I. R. Innes. 1985. Norepinephrine on venous compliance and unstressed volume in cat liver. Am. J. Physiol. 248:H468-H476.

19. Stokland, O., M. M. Miller, A. Ilebekk, and F. Kiil. 1980. Mechanism of hemodynamic responses to occlusion of the descending thoracic aorta. Am. J. Physiol. 238:H423-429.

20. Stokland, O., J. Thorvaldson, A. Ilebekk, and F. Kiil. 1982. Contribution of blood drainage from the liver, spleen and intestines to cardiac output of aortic occlusion in the dog. Acta Physiol. Scand. 114:351-362.

21. Gaddis, M. L., C. F. Rothe, R. S. Tunin, M. Moran, and C. L. MacAnespie. 1986. Mean circulatory filling pressure: potential problems with measurement. Am. J. Physiol. 251:H857-H862.

22. Caldini, P., S. Permutt, J. A. Waddel, and R. L. Riley. 1974. Effect of epinephrine on pressure, flow, and volume relationship in the systemic circulation in dogs. Circ. Res. 34:606-623.

23. Green, J. F. 1977. Mechanism of action of isoproterenol on venous return. Am. J. Physiol. 232:H152-H156.

24. Engler, R. L., G. L. Freeman, and J. W. Covell. 1983. Regional venous return: nitroprusside effect in normal and chronically congested dogs. Am. J. Physiol. 245:H814-H828. 